e las eras a los paisajes. Una perspectiva crítica sobre la «era del átomo»: encuentro entre CTS y filosofía de la tecnología

From Ages to Landscapes. A Critical Perspective on the "Age of the Atom": Encounters between STS and Philosophy of Technology

(ID) Bernadette Bensaude-Vincent ${ }^{*}$

\title{
(2) $(\mathbb{Q} \otimes$
}

" Profesora emérita de la Université Paris 1 Panthéon-Sorbonne, Francia. Correo electrónico: bernadette.bensaude-vincent@univ-paris1.fr

Fecha de recepción: 14 de septiembre de 2020

Fecha de aceptación: 24 de febrero de 2021

\section{Cómo referenciar / How to cite}

Bensaude-Vincent, B. (2021). De las eras a los paisajes. Una perspectiva crítica sobre la «era del átomo»: encuentro entre CTS y filosofía de la tecnología. Trilogía Ciencia Tecnología Sociedad, v. 13, n. 25, e1760. https: / / doi.org/10.22430/21457778.1760 
Resumen: los estudios CTS han intentado que la ciencia observe todo lo que ocurre en un campo de estudio y que no se limite a la teoría, como se evidencia en lo que se llama el «giro empírico» en la filosofía de la tecnología. Este estudio muestra el posible vínculo entre la filosofía de la tecnología y la perspectiva CTS, y propone que el concepto de tiempo-paisaje es más apropiado que el de tiempo cronológico para entender los cambios tecnológicos actuales. En este sentido, más allá de las conjeturas teóricas sobre la tecnología, esta investigación toma la energía nuclear como caso de estudio para cuestionar y ponderar los postulados tradicionales del cambio técnico, adoptando el principio metodológico de la exigencia de concreción de los estudios empíricos y el concepto de imaginario sociotécnico. El proceso de indagación combinó elementos como la epistemología, la ética y la política en una perspectiva antropológica. Finalmente, se establece que el uso de la energía nuclear, considerada como parte de la era del átomo, invita a pensar en el tiempo en términos distintos a los cronológicos. El estudio también ayuda a ilustrar los beneficios mutuos de una asociación entre los estudios CTS y la filosofía de la tecnología.

Palabras clave: Antropoceno, residuos nucleares, imaginario sociotécnico, filosofía de campo.

Abstract: STS studies have sought to make science look at everything going on in a field of study and not limit itself to theory, as evidenced by what is called the "empirical turn" in the philosophy of technology. This study shows the possible link between the philosophy of technology and the STS perspective, proposing the concept of time-landscape as more appropriate than that of chronological time to understand current technological changes. In this sense, beyond the theoretical conjectures about technology, this research takes nuclear energy as a case study to question and weigh the traditional assumptions of technical change, adopting the methodological principle of the requirement of concreteness of empirical studies and the concept of socio-technical imaginary. The inquiry process combined elements such as epistemology, ethics and politics in an anthropological perspective. Finally, it is argued that the use of nuclear energy, considered as part of the age of the atom, opens the possibility of thinking about time in terms other 
than chronological. The study also helps to illustrate the mutual benefits of a partnership between STS studies and philosophy of technology.

Keywords: Anthropocene, nuclear waste, sociotechnical imaginary, fieldwork philosophy. 
De las eras a los paisajes. Una perspectiva Crítica Sobre la «ERA Del átomo»: encuentro entre CTS y filosofía de la TECNOLOGía

\section{INTRODUCCIÓN}

Tratando de promover el concepto de tiempo-paisaje como más apropiado que el de tiempo cronológico para pensar en los cambios tecnológicos y planetarios actuales, este artículo ilustra una posible intersección entre la filosofía de la tecnología y la perspectiva CTS. De la tradición CTS adopta el principio metodológico de la exigencia de concreción, de los estudios empíricos y el concepto de imaginario sociotécnico. Los CTS han enseñado a ver la ciencia en acción y a observar todo lo que ocurre en un campo de estudio (Latour, 1987). Este legado de la teoría del actor-red (TAR) y de su homóloga estadounidense, la construcción social de la tecnología (SCOT, por sus siglas en inglés), (Pinch \& Bijker, 1984; Bijker et al., 1987) está presente en el llamado «giro empírico» de la filosofía de la tecnología (Brey, 2010), especialmente en Verbeek (2005, 2008), Feenberg $(2000,2003)$ y Mol (2002). En lugar de especular sobre la técnica en general, este artículo parte de un estudio de caso, la energía nuclear, para cuestionar y poner a prueba las descripciones tradicionales de la historia y de la filosofía de las técnicas. Esta práctica empírica de la filosofía, que este estudio denomina «filosofía de campo», combina íntimamente la epistemología, la ética y la política en una perspectiva antropológica.

El concepto de imaginario sociotécnico designa una construcción histórica a mitad de camino entre el mundo concreto y la abstracción intelectual que conforma las estructuras sociales y la acción humana. Jasanoff y Kim (2015) lo definen como «visiones colectivas, institucionalmente estabilizadas y públicamente representadas de futuros deseables, animadas por la comprensión compartida de formas de vida y de orden social que se pueden alcanzar a través de los avances de la ciencia y la tecnología» (p. 4). Si se asume que las visiones del futuro no pueden aislarse de las del pasado y el presente, este artículo amplía la noción de imaginario sociotécnico a la totalidad del tiempo. El tiempo solo se piensa a través de metáforas: el curso del tiempo, la flecha del tiempo, la escala del tiempo son los más conocidos. Están tan arraigados en la cultura que ni siquiera se piensan y pasan por realidades. Porque las metáforas son mucho más que recursos retóricos. Son altamente performativos y dan forma a nuestra experiencia del mundo (Austin, 1962; Lakoff \& Johnson, 1980). Crean un orden del tiempo, una articulación específica de pasado, presente y futuro que constituye lo que el historiador Hartog (2015) llama un «régimen de historicidad». 
El esfuerzo por problematizar el tiempo lineal y universal, una noción común y evidente, sitúa este artículo sobre todo en la tradición filosófica francesa, ejemplificada por Michel Serres, Michel Foucault y Gilles Deleuze. Más allá de la diferencia entre sus estilos, iniciaron colectivamente una tendencia que dio un vuelco a las categorías filosóficas del pensamiento moderno y promovió nuevos conceptos. Con fundamento en análisis históricos, su trabajo se abre, sin embargo, al mundo y a los problemas políticos de su tiempo. Desde el punto de vista de la filosofía de la técnica, este trabajo se basa en pensadores franceses como François Dagognet y Gilbert Simondon, que dieron a los objetos un lugar en la filosofía (Loeve et al., 2018). La tradición que iniciaron Serres, Foucault y Deleuze se extiende hoy en día en un espacio teórico que se sitúa en un cruce entre CTS y la filosofía, ocupado por autores como Bruno Latour, Isabelle Stengers o Donna Haraway, y otros tantos que se esfuerzan por pensar la crisis ecológica renovando las categorías del pensamiento. Una constelación de autores que el diario Le Monde presentaba hace poco como los «ecosofistas» (Truong, 2020), porque se interesan sobre todo por la crisis climática, sus aspectos antropológicos y políticos, y participan de las discusiones sobre el Antropoceno, esta nueva era de la historia de la Tierra en la que la humanidad se convierte en una fuerza geológica.

Para caracterizar de manera muy esquemática ese cuadro filosófico que Le Monde califica de «nueva ola», este artículo conservará el rechazo de tres categorías estructurantes del pensamiento occidental ilustrado por Descartes y Kant: la distinción entre sujeto y objeto, la división entre naturaleza y cultura y, por último, el excepcionalismo humano. Así, se perfila una ontología que rompe con la metafísica cartesiana y kantiana.

Sin embargo, esta nueva ontología solo se separa parcialmente de la metafísica clásica, puesto que no cuestiona la hegemonía del tiempo universal y lineal de la cronología. Mientras que el prefijo «anthropos» del Antropoceno ha suscitado muchas objeciones y todo tipo de neologismos como «capitaloceno» o «chtuluceno», el «ceno», es decir, el postulado de una era que se inscribe en la cronología de la Tierra permanece intacto. Se debe ir más allá: la supremacía del tiempo cronológico oculta otras perspectivas sobre el tiempo, que podrían ser más pertinentes para tratar los problemas del Antropoceno.

Una alternativa a la flecha del tiempo es la metáfora del tiempo-paisaje. Liberarse de la línea cronológica que franquea las eras a golpe de potencias de diez para 
De las eras a los paisajes. Una perspectiva Crítica Sobre la «ERA Del Átomo»: encuentro entre CTS y Filosofía de la TECNOLOGÍA

definir las escalas de tiempo es abrir los ojos a las temporalidades propias de las cosas que nos rodean y a los vínculos de interdependencia que crean nuestras elecciones tecnológicas. Ese tiempo-paisaje (timescape en inglés) queda definido por Adam (1998) así:

Con la idea de tiempo-paisaje busco una extensión de la perspectiva del paisaje, es decir, desarrollar una receptividad análoga a las interdependencias y ausencias temporales, y captar los fenómenos ambientales como un todo temporal complejo y contextualmente específico. Esto requiere un cambio en el énfasis, no solo del espacio al tiempo, sino, aún más importante, a eso que es invisible y que está por fuera de la capacidad de nuestros sentidos (p. 9).

Un tiempo-paisaje está constituido por la interacción de entidades antropogénicas y naturales (Bensaude-Vincent, 2021). Se trata de un lugar de vida y no solo una escena pintoresca que contempla un observador externo. Un recorrido paisajístico por las sucesivas edades de la civilización, y la técnica es un buen ejercicio para deshacer la íntima asociación entre técnica y progreso que alimenta la flecha del tiempo. Este artículo trata de la era del átomo porque, después de muchos debates, el grupo de expertos encargados de definir la fecha del comienzo del Antropoceno señala las primeras explosiones nucleares atómicas como el mejor marcador estratigráfico (Lewis \& Maslin, 2015).

El artículo comienza con una breve presentación del imaginario sociotécnico que define la era del átomo. La segunda parte problematiza la noción de era, o de paradigma técnico, actualizando los supuestos implícitos en esta categoría tradicional vinculada con otras expresiones comunes en la historia de la técnica. La tercera parte toma un desvió filosófico que presenta de manera sucinta los puntos de ruptura entre la filosofía moderna y la nueva ontología emergente, en particular en los estudios sobre el Antropoceno. Por último, en la cuarta y quinta partes se expone un enfoque alternativo a la era del átomo bajo la forma de un tiempo-paisaje, fundando en la cercanía entre tiempo y clima y prestando atención a las cosas más que a los humanos. 


\section{La era del átomo}

El 6 de agosto de 1945, un B-29 de la Fuerza Aérea de los Estados Unidos lanza una bomba atómica sobre Hiroshima. Harry S. Truman anuncia ese bombardeo en la radio como el amanecer de una nueva era de abundancia que exige esfuerzos de investigación:

El hecho de que podamos liberar energía atómica inaugura una nueva era en la comprensión de las fuerzas de la naturaleza por parte del hombre. En el futuro, la energía atómica podrá complementar la que ahora viene del carbón, el petróleo y el agua, pero que por el momento no se puede producir de manera que pueda competir comercialmente con esas fuentes. Antes de que eso suceda, debe darse un largo periodo de intensa investigación. Nunca ha sido costumbre de los científicos de este país ni la política de este gobierno esconder al mundo el conocimiento científico. En consecuencia, la norma será hacer público todo acerca del trabajo con la energía atómica (American Experience, 1945, párr. 13).

En Francia, Camus (1945) protestó contra las celebraciones de este acontecimiento en la prensa internacional y propuso otra visión:

La civilización mecánica acaba de alcanzar su último grado de salvajismo. Tendremos que escoger, en un futuro más o menos próximo, entre el suicidio colectivo o la utilización inteligente de las conquistas científicas (párr. 1).

Muchos intelectuales internacionales ven Hiroshima como una catástrofe, un suicidio colectivo. La perspectiva del fin de los tiempos despierta las especulaciones milenaristas. En octubre, Sartre (1945) escribe en el primer número de Temps Modernes: «Henos aquí (...) en el año 1000, cada mañana estaremos en la víspera del fin de los tiempos». Charbonneau (2014), filósofo protestante, da una conferencia titulada «L'An 2000», en la que presenta la bomba atómica como un salto adelante de algunos miles de años. El historiador norteamericano Mumford (1946) hace un llamado para presionar a los políticos: «Caballeros: ustedes están locos».

Los locos están planeando el fin del mundo. Lo que llaman progreso continuo en la guerra atómica no es más que el exterminio universal, y lo que llaman seguridad nacional es el suicidio organizado. Solo tenemos una obligación en este momento: 
De las eras a los paisajes. Una perspectiva Crítica Sobre la «ERA Del Átomo»: encuentro entre CTS y Filosofía de la TECNOLOGÍA

cualquier otra tarea es un sueño y una burla. Detengan la bomba atómica. Detengan la construcción de la bomba. Abandonen la bomba completamente. Desmantelen cualquier bomba existente (p. 5).

La coexistencia de estos puntos de vista opuestos sugiere que el evento se interpretó en un marco religioso. En la Biblia, el libro del Apocalipsis es un momento de revelación que desvela tanto el fin de los tiempos como un mundo celestial en el que el lobo vivirá con el cordero.

El primer número del Bulletin of Atomic Scientists, de 1947, publicó una imagen de un reloj con la aguja puesta siete minutos antes de la medianoche, creando un fuerte vínculo entre las imágenes medievales del Juicio Final y el tic-tac de la bomba. ${ }^{1}$ Hiroshima aparece como un acontecimiento sin precedentes que rompe la continuidad de la experiencia humana, creando una sensación de desconexión entre el pasado y el futuro. El futuro ya no se desprende del estado de cosas del ayer. El mundo después de Hiroshima ya no será el mismo que era ayer. Pese a sus diferencias, los apóstoles de un futuro brillante y los profetas de un apocalipsis nuclear señalan a Hiroshima como un punto de inflexión en la historia del mundo, el comienzo de la era del átomo. Y todos ellos apoyan la visión occidental de una flecha del tiempo. Poco importa que la flecha apunte hacia un mundo mejor o hacia la catástrofe: el tiempo es único, lineal y dirigido.

El término «era del átomo» se remonta a 1945 y gana popularidad en la década de 1950, cuando las campañas de «Átomos para la Paz» proclamaban que el átomo podía utilizarse con fines pacíficos y productivos, que podía proporcionar electricidad gratuita, combustible para cohetes espaciales o automóviles, conservantes de alimentos y tratamientos contra el cáncer. Incluso Japón, víctima de dos bombas atómicas, construye gustosamente reactores nucleares. Para los japoneses, incluidos los supervivientes de las dos bombas, la energía nuclear encierra la promesa de la reconstrucción, el progreso y el confort modernos (Zwigenberg, 2015). En todos los países nucleares, el átomo es una garantía de independencia y soberanía.

Por supuesto, la energía nuclear ha sido cuestionada desde el principio, excepto en sus aplicaciones médicas. Los opositores a las armas nucleares nunca cesaron sus acciones y manifestaciones, e Hiroshima se convirtió en el centro mundial

${ }^{1}$ Sin embargo, muchos de estos físicos atómicos, Frédéric Joliot-Curie en particular, estaban a favor de los usos pacíficos del átomo y contribuyeron activamente al desarrollo de los reactores nucleares (Pinault, 2000). 
de todos los movimientos de desarme. Pero hasta los años 70, la protesta se refería exclusivamente a las armas atómicas. Las campañas contra las pruebas nucleares no afectan al sueño nuclear y se construyen decenas de reactores en países industrializados como Estados Unidos, la Unión Soviética, el Reino Unido, Japón y Francia. La era atómica se celebra como una etapa de la civilización, comparable a la Edad de Bronce o la Edad de Hierro, un paso decisivo en la «Marcha de la Humanidad», el largo proceso de emancipación de la humanidad ante la naturaleza.

En los años 80, las promesas de la era atómica comenzaron a tambalearse. El orden nuclear posterior a Hiroshima se basa en una división entre armas nucleares y energía, entre destrucción y construcción. Pero una serie de catástrofes (Isla de las Tres Millas en 1979; Chernóbil en 1986) han revelado el lado más oscuro de la energía nuclear civil. Confirman los temores previos de la opinión pública sobre los reactores nucleares, ya que la película El síndrome de China, estrenada unos días antes del accidente de la Isla de las Tres Millas, muestra el escenario de una fusión del núcleo. Las facetas oscuras de la energía nuclear civil difuminan la línea entre los «buenos» y los «malos», entre el átomo agresivo y el pacífico. A pesar de que el Organismo Internacional de Energía Atómica ha establecido un régimen exhaustivo de control, evaluación de riesgos y regulación, surgen dudas sobre la seguridad de las plantas y minas de uranio, y crece la protesta pública. El accidente de Chernóbil demuestra además que la opinión de los expertos no siempre es creíble (Kuchinskaya, 2014). Para combatir los puntos ciegos y la ignorancia social, se crean redes de contraexperiencia, como el CRIIRAD en Francia (Topçu, 2013). El accidente de Fukushima en 2011 revela la vulnerabilidad de las instalaciones: un terremoto, seguido de un tsunami, superaron las medidas de seguridad. Ni la movilización de expertos ni la revisión de las normas y pruebas tras la catástrofe pudieron frenar la oleada de críticas y dudas. La era del átomo se ha convertido en una era de escepticismo y sospecha. Además de los daños a largo plazo para la salud y el medio ambiente, hay problemas con el desmantelamiento de las centrales nucleares de primera generación y la gestión de un volumen cada vez mayor de residuos nucleares peligrosos. Así, en pocas décadas, el imaginario de la era atómica dio un vuelco: del entusiasmo a la desconfianza, de las promesas de progreso a los daños y amenazas permanentes. La energía nuclear se muestra como una tecnología envejecida, próxima a desaparecer. Es muy significativo que, en Francia, el CEA (Comisión de Energía Atómica), creado en 1945, haya pasado a llamarse Comisión de Enetgía Atómica y de Energías Alternativas. 
De las eras a los paisajes. Una perspectiva Crítica Sobre la «ERA Del Átomo»: encuentro entre CTS y Filosofía de la TECNOLOGÍA

\section{Lo impensable de los paradigmas tecnológicos}

De la cima al declive es la narrativa común que se encuentra en toda la era del átomo. Es una visión relativamente buena y fiel de un periodo abierto por un acontecimiento importante y que llega a su fin por la aparición de nuevas energías. Por supuesto, podemos discutir las fechas de inicio y finalización: la mayoría de los relatos eligen el 16 de julio de 1945, fecha de la prueba Trinity en Nuevo México, mientras que esta primera explosión atómica, al ser secreta, no pudo ser un acontecimiento. Del mismo modo, el fin de la era del átomo no es inminente ni seguro ya que, en el contexto del cambio climático, la energía nuclear se ofrece como una fuente de energía limpia y sin emisiones de carbono que puede salvar el planeta en lugar de destruirlo.

Pero el problema de narrativas como la de la era del átomo no radica tanto en la fijación de fechas que delimitan el periodo, como en la suposición tácita de que la historia se compone de una secuencia de fases distintas caracterizadas por un patrón dominante. Se supone que las técnicas se desarrollan según modelos sucesivos, análogos a los paradigmas científicos de Thomas Kuhn. Se caracterizan por un conjunto de problemas que resolver, que encuentran su solución superando obstáculos y dificultades hasta que otro paradigma los supera.

La flecha del tiempo, jalonada de acontecimientos significativos, se impone como un hecho evidente que nunca se cuestiona. Subyace a la práctica común de distinguir fases en el desarrollo de la civilización, bien por el dominio de un material (eras de la piedra, del bronce, del hierro), bien por el dominio de una fuente de energía (eras del carbón, del petróleo, del átomo). Y en el lenguaje cotidiano utilizamos expresiones como «la era de la información», «la revolución digital», «la transición energética». La metáfora de la flecha del tiempo está tan arraigada en nuestra cultura que nos parece evidente que la historia se desarrolla en un solo hilo, que los cambios tecnológicos tienen un impacto masivo y global que está cambiando la faz del mundo. Los debates entre los especialistas se centran únicamente en la continuidad o discontinuidad de los cambios.

Mientras los historiadores se centren en el cambio tecnológico, mantendrán la ilusión de que una nueva tecnología está desplazando a las anteriores. El sesgo proviene de la fascinación por la novedad. Por otro lado, los historiadores de la tecnología, como Hård y Jamison $(1998,2005)$ o Edgerton (2007), que se centran 
en la vida de la tecnología más que en las innovaciones, muestran que lo antiguo coexiste con lo nuevo. $\mathrm{Y}$ nos recuerdan que, a finales del siglo $\mathrm{XX}$, una parte importante de la humanidad no tenía acceso al agua o a la electricidad; que la píldora anticonceptiva no ha expulsado al preservativo. Cuando hablamos de cambios de paradigma, no vemos que en realidad estamos evolucionando en una mezcla tecnológica de lo viejo con lo nuevo. En nuestra vida cotidiana aún se utilizan técnicas tan antiguas como el soplado de vidrio, la fabricación de acero, la fermentación, etc. La impresión de novedad radical se contradice con los hechos y las cifras. Una nueva fuente de energía no sustituye a la antigua. La energía hidroeléctrica y la termoeléctrica sobreviven a la nuclear. Como muestra Fressoz (2014), no existe una verdadera transición energética, sino que una nueva energía se añade a las anteriores. Hoy se consume más carbón que en el siglo XIX, y las energías renovables no han frenado la exploración y extracción de gas y petróleo. El Antropoceno es el resultado de esta acumulación y no de un cambio de paradigma iniciado en 1945.

Por lo tanto, si nuestra época es una mezcla tecnológica antes que un paradigma, debemos cuestionar las grandes narrativas sobre las edades de la humanidad, porque se basan en el postulado del curso uniforme de un tiempo único y universal que puede dividirse en épocas por los momentos decisivos. Abordar el tiempo cronológico lineal es un proyecto ambicioso que requiere un desvío filosófico, una mirada al legado de la crítica de la modernidad iniciada por Serres, Foucault y Deleuze.

\section{La deconstrucción de la ontología moderna}

Estos filósofos prepararon el camino para esta crítica de la hegemonía del tiempo cronológico al deconstruir categorías fundamentales como los dualismos sujeto / objeto y naturaleza / cultura y al abrir el espacio a una crítica del estatus excepcional de la humanidad.

La distinción entre sujeto y objeto es el fundamento de la ciencia moderna, que subordina el conocimiento de la realidad a la objetividad y subyace al proyecto cartesiano de «hacerse dueño y señor de la naturaleza» (Descartes, 1637). Esta distinción, que por mucho tiempo se consideró como evidente, fue socavada por primera vez por Foucault (1966) en su famosa obra sobre el cuadro de Velásquez, 
De las eras a los paisajes. Una perspectiva Crítica Sobre la «ERA Del Átomo»: encuentro entre CTS y Filosofía de la TECNOLOGía

Las meninas, que sugiere que la perspectiva del soberano puede intercambiarse con otras. Y se ha vuelto aún más problemática a la luz del trabajo de la epistemología histórica y el giro hacia la práctica en los Science Studies. Por un lado, las nociones de objetividad y verdad son construcciones históricas que han forjado las dos entidades metafísicas de sujeto y objeto (Daston \& Galison, 2007). Por otra parte, el conocimiento científico no puede reducirse a un encuentro cara a cara entre el sujeto y el objeto, ya que implica a un conjunto de actores: instrumentos, publicaciones, instituciones, inversiones y los propios objetos que participan en la construcción del conocimiento (Latour, 1987; Hacking, 1983).

La división entre naturaleza y cultura (o sociedad), fundamento de todo el pensamiento occidental desde la antigua Grecia, es uno de los objetivos favoritos de Michel Serres. En Le passage du Nord-Ouest, Serres (1980), critica abiertamente la división del conocimiento entre las ciencias naturales y las humanas. Para producir nuevos conocimientos e innovaciones técnicas, debemos atrevernos a desafiar esta barrera y las divisiones disciplinares para aventurarnos en una navegación arriesgada, llena de escollos, pero rica en promesas y novedades. En Le contrat naturel, Serres (1990) demuestra que la división entre naturaleza y sociedad, que dio lugar a la noción de contrato social, establece una guerra contra la naturaleza y propone un nuevo derecho basado en la interdependencia de las sociedades humanas con la naturaleza. Latour $(1991,1999)$ desarrolla la crítica de un modo más antropológico en Nous n'avons jamais été modernes y más político en Politiques de la nature, que esboza una nueva ecología política.

Vinculada a las dos divisiones anteriores, la posición excepcional de la humanidad sustenta el humanismo clásico y los derechos humanos, así como la idea kantiana de una historia universal (Kant, 1784). La crítica al excepcionalismo humano no es un tema central en las obras de Serres, Foucault o Deleuze. Más bien procede de la filosofía medioambiental iniciada por Aldo Leopold, que opone al antropocentrismo perspectivas biocéntricas o ecocéntricas e invita a una mayor empatía con los demás habitantes del planeta. Los defensores de la ética y los derechos de los animales, como Singer (2012), cuestionan la idea de que los humanos merecen más cuidados y respeto que otros animales. En antropología, Haraway (2007) critica sistemáticamente las demarcaciones entre humanos y no humanos, especialmente entre especies animales. Por último, la ecología política pide que se preste más atención a la diversidad de seres vivos vulnerables, tanto humanos como no humanos (Srinivasan \& Kasturirangan, 2016). De la 
convergencia de estas diversas corrientes resulta algo así como una «ontología plana», que trata a los seres naturales y sociales, a las cosas y a las personas, como actores iguales en la historia.

Esta ontología que transgrede los dualismos se ve reforzada por la noción de Antropoceno, proveniente de las ciencias del Sistema Tierra. Se trata de una nueva gran narrativa en la que los seres humanos se han convertido en la fuerza dominante que moldea la composición y los procesos biogeofísicos del planeta (Crutzen \& Stoemer, 2000). Poner de relieve el impacto de la historia sociotécnica en la historia de la Tierra saca al ser humano de su aislamiento. Los investigadores de ciencias sociales y humanidades no tardaron en darse cuenta del alcance de las consecuencias que esto podía tener en sus disciplinas. Se subieron al carro de los estudios del Antropoceno hasta el punto de que su número de publicaciones sobre el tema rivaliza con el de los científicos naturales (Linnér \& Wibeck, 2015). Entre los pioneros, Chakrabarty (2009), historiador del poscolonialismo, señala que el Antropoceno arruina la distinción entre dos categorías de ciencias, la del hombre y la de la naturaleza. Latour $(2014,2017)$ encuentra en el Antropoceno una confirmación de sus tesis sobre la modernidad, ya que socava la idea de una naturaleza externa al hombre y la idea corolario de sujetos autónomos situados ante una naturaleza objetiva. Por lo tanto, ve en este «nuevo régimen climático» una oportunidad para promover su proyecto cosmopolítico. Para el antropólogo Descola (2018a, 2018b), el Antropoceno supone el fin de la hegemonía de la ontología occidental de la naturaleza y reclama una ontología pluralista.

\section{El desafío a la hegemonía del tiempo cronológico}

Muchos científicos sociales han criticado el Antropoceno por mantener una ontología fundamentalmente antropocéntrica que hace del antropos, un sujeto colectivo y abstracto, totalmente descontextualizado, el responsable de los trastornos climáticos (Moore, 2016; Bonneuil \& Fressoz, 2012). Pero rara vez se cuestiona la propuesta de una nueva era geológica.

$\mathrm{Y}$, sin embargo, moviliza las escalas del tiempo geológico con sus subdivisiones jerárquicas: eón, era, periodo, época, suelo y subsuelo. Sin duda, los debates sobre el inicio del Antropoceno -1492 (descubrimiento del Nuevo Mundo), 1784 (máquina de vapor) o 1954 (pruebas nucleares) - influyen en la duración de esta 
De las eras a los paisajes. Una perspectiva Crítica Sobre la «ERA Del Átomo»: encuentro entre CTS y Filosofía de la TECNOLOGÍA

nueva era. Pero estos debates nunca cuestionan la legitimidad de considerar el tiempo con potencias de diez (Hooft \& Vandoren, 2014). Por el contrario, estos debates son una forma de revitalizar las escalas temporales geológicas. Un protagonista del debate, Michael Walker (citado en Monastersky, 2015), señala: «En mi opinión, la escala de tiempo geológica es uno de los grandes logros de la humanidad» (p. 145). Al subrayar la colusión entre las potencias de diez, el «tiempo profundo» de la historia de la Tierra y el tiempo corto de la historia sociocultural, el Antropoceno refuerza la pertinencia de una escala temporal única en la que se alinean los fenómenos cosmológicos y geológicos que tienen lugar a lo largo de millones de años, la evolución biológica que se extiende a lo largo de algunos millones o cientos de miles de años, y los fenómenos históricos que se cuentan en cientos o incluso decenas de años.

Sí, la escala de tiempo es «uno de los grandes descubrimientos de la humanidad», pues permite a los científicos medir con cierta precisión la edad del universo, así como las escasas fracciones de segundo de la vida de las partículas subatómicas. Pero al hacer conmensurables todos los tiempos de esta manera, ordenándolos por potencias de diez en la misma línea cronológica, necesariamente descuidamos el hecho de que puede haber regímenes temporales diversificados, cíclicos, lineales, caóticos... Adquirimos el privilegio de la conmensurabilidad a costa de cegarnos ante la variedad y heterogeneidad de los tiempos de la naturaleza, así como de la cultura (Bouton \& Huneman, 2017; Baschet, 2018). Las potencias de diez producen un efecto pantalla sobre los posibles conflictos de temporalidad entre los fenómenos en curso en nuestro planeta.

La construcción del tiempo cronológico se basa en la suposición tácita de que todos los fenómenos siguen la misma trayectoria uniforme y que solo difieren en su velocidad. Para esclarecer este punto, la música nos brinda una distinción útil entre ritmo y tempo. El vals tiene un ritmo de tres tiempos, que se toca con un tempo lento (largo o adagio) o rápido (allegro vivace o prestissimo). Como lo recuerda Serres (2019), nunca será un «vals de mil tiempos» (p. 81), pese a la bella canción de Jacques Brel, pues el tiempo cronológico solo conoce los golpes del cronómetro. Solo ellos se tienen en cuenta en la gran narrativa del Antropoceno, que señala las variaciones del tempo de la danza en el planeta.

El argumento más sólido en apoyo de la introducción de un nuevo «ceno» es una colección de curvas ampliamente difundidas en las publicaciones científicas y entre el público con el título de «La gran aceleración» (ver Figura 1). 


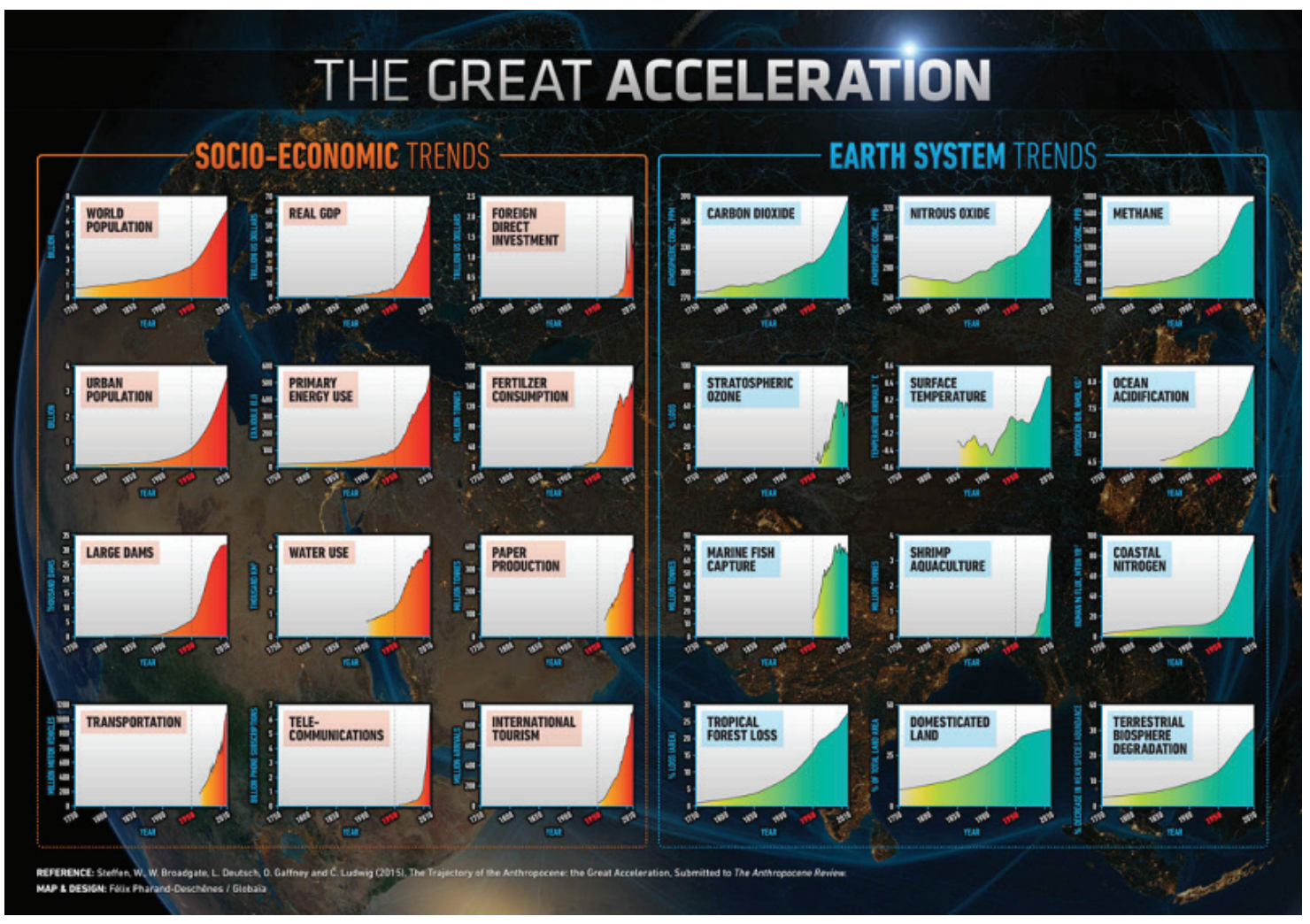

Fuente: International Geophere-Biosphere Programme (2015).

Esta colección de 24 gráficos, que se ha convertido en el emblema del Antropoceno, compara la evolución de doce fenómenos socioeconómicos (crecimiento demográfico, crecimiento económico, transporte, etc.) con la de doce fenómenos relacionados con el sistema Tierra (concentración de gases de efecto invernadero en la atmósfera, temperaturas de la superficie, acidificación de los océanos, etc.). Esta colección ilumina el paralelismo de las curvas de los indicadores biogeológicos y socioeconómicos. $\mathrm{El}$ aumento del $\mathrm{CO}_{2}$ sigue la misma pendiente que el consumo de energía y del Producto Interno Bruto (PIB), indicador del crecimiento económico. El nervio de la prueba reside, por tanto, en reunir fenómenos heterogéneos. Aunque la correlación no implica causalidad, concluyen Steffen et al. (2015), el paralelismo es tan sorprendente que deja claro que no se trata de fenómenos independientes.

En poco más de dos generaciones (o una sola vida) la humanidad (o una pequeña fracción de ella) se ha convertido en una fuerza geológica a escala planetaria. 
De las eras a los paisajes. Una perspectiva Crítica Sobre la «ERA Del Átomo»: encuentro entre CTS y Filosofía de la TECNOLOGÍA

Hasta ahora, las actividades humanas eran insignificantes en comparación con el sistema biofísico de la Tierra, y ambos podían funcionar de forma independiente. Sin embargo, ahora es imposible considerar una cosa separada de la otra (p. 94).

La demostración desemboca en la siguiente alternativa: ¿Gran desacoplamiento o gran colapso en los próximos 50 años? Y los autores recomiendan un planetary stewardship. Sin duda, se trata de erigirnos en guardianes más que en dueños y señores de la naturaleza, pero el Antropoceno conserva el excepcionalismo de la ontología moderna. La singularidad de la humanidad sale en todo caso reforzada por la misión de guardián que se le confiere (Conty, 2016).

¿Cómo comprender que el excepcionalismo humano resiste a la evidencia de las interdependencias entre historia humana e historia de la Tierra? Y es que la singularidad humana se postula en la propia construcción del tiempo cronológico universal. Para construir una cronología que abarque tanto los fenómenos a escala cósmica como a escala molecular, hay que situarse necesariamente fuera del universo, fuera del tiempo. El tiempo global universal presupone una visión de la nada, o más bien del no tiempo. De ahí la ambivalencia del Antropoceno, que subraya la interdependencia global y sigue siendo decididamente antropoexcepcionalista.

\section{Tiempo y clima}

La gran narrativa del Antropoceno, basada en el postulado del tiempo cronológico universal, no afecta a la flecha del tiempo. No más que las grandes narraciones de la era atómica, con su característica oscilación entre el progreso y la catástrofe. Ambos imaginarios confieren a los humanos un estatuto excepcional en el mundo. Sin embargo, por varias razones, la tecnología nuclear, el supuesto desencadenante del Antropoceno, puede sugerir otra visión del tiempo menos antropocéntrica y menos lineal.

La primera razón es que ha provocado un relacionamiento entre el paso del tiempo y el clima. La relación entre el tiempo y el clima se hizo inmediatamente. Mientras se hablaba del apocalipsis y del suicidio masivo en la década de 1950, los militares estadounidenses y los expertos de la RAND Corporation estudiaban el impacto de las bombas de Hiroshima y Nagasaki en el clima de Japón. Pero como 
esos estudios eran secreto de defensa, esa relación no se debatió públicamente (Badash, 2009; Dörries, 2011). De ahí la llamativa discrepancia con el imaginario de la era atómica, centrado exclusivamente en el destino de los humanos, como autores y víctimas de la historia. El apocalipsis no se debe a los pecados de la humanidad en general, sino a un grupo de individuos, los locos o Estados canallas con armas atómicas, que son los únicos que tienen las claves del futuro (Weart, 1992). Además, el apocalipsis nuclear significa el fin de la humanidad. El futuro del planeta y de los seres vivos queda fuera de la vista.

No fue hasta 1982 cuando Paul J. Crutzen, el químico atmosférico que acuñaría la noción de Antropoceno, publicó un artículo con John W. Birks, que describe el impacto de la lluvia atómica en el clima. Basándose en simulaciones computarizadas, calculan que los incendios forestales provocados por la explosión de una bomba $\mathrm{H}$ producirían un humo tan denso que reduciría drásticamente la radiación solar en la superficie de la Tierra, lo que provocaría un descenso de las temperaturas globales (Crutzen \& Birks, 1982). El escenario del invierno nuclear que asocia el imaginario del apocalipsis con el ciclo de las estaciones cambia la visión del tiempo. La historia de la humanidad deja de estar separada de la historia de la Tierra. Entre 1982 y 1985, este escenario desencadenó una polémica liderada por el popularísimo Carl Sagan, que llevó el tema a la esfera política con el senador Albert Gore. En la URSS, el escenario alimenta una campaña contra el imperialismo estadounidense. Aunque el debate se centra únicamente en las armas nucleares, acerca por primera vez el tiempo (time o Zeit) y el clima (weather or Weter).

La conexión es evidente en las lenguas latinas; el francés, el español y el portugués tienen una sola palabra para ambas cosas: temps, tiempo, tempo. Serres (1994) ha subrayado cómo esta comparación entre el tiempo de los meteoros y el tiempo medido por los relojes ha parecido durante mucho tiempo injustificada. Primeramente, por un fuerte contraste: entre el mundo celeste y el mundo sublunar de Aristóteles y más tarde entre las rigurosas previsiones de la mecánica y la imprevisibilidad de los meteoros. Los calendarios, almanaques y horarios, todas las infraestructuras que permiten controlar el tiempo, se basan en unidades de tiempo fijadas a partir de la astronomía y, desde 1967, de la física atómica. La medición cronológica del tiempo se basa en fenómenos que obedecen a leyes rigurosamente deterministas, mientras que el clima depende de fenómenos complejos y caóticos y de acontecimientos cataclísmicos. 
De las eras a los paisajes. Una Perspectiva Crítica sobre la «ERa del Átomo»: encuentro entre CTS y Filosofía de la TECNOLOGía

Pero la relación entre el tiempo y el clima, esbozada por el escenario del invierno nuclear, se ha intensificado bajo la presión de las actuales perturbaciones climáticas. A la amenaza de un apocalipsis se añade el temor a un colapso de las infraestructuras económicas y sociales que conduzca al fin de la civilización. El colapso o colapsismo es un movimiento de moda, muy visible en todos los medios de comunicación. Aunque la flecha del tiempo sigue dominando la imaginación, la visión es menos antropocéntrica. Se extiende a todos los seres vivos, a la biodiversidad. Sobre todo, la catástrofe ya no es un acontecimiento aislado que marque el punto de inflexión de una época. Es un proceso lento y gradual, desencadenado por el capitalismo, la industrialización y no solo por la energía nuclear. Es un proceso que hay que vivir y gestionar. Es llamativo ver cómo la literatura colapsista se interesa más por el tiempo que por el fin de los tiempos (Danowski \& Viveiros de Castro, 2016). Se trata de ajustar nuestro comportamiento, ya sea para frenar el proceso o para acelerarlo con el fin de precipitar el colapso del capitalismo. Pues la imaginación colapsista forja un tiempo que ya no es del todo lineal en la medida en que da rienda suelta a las visiones de un mundo sin nosotros, un mundo esclavizado y libre de depredadores humanos, o un mundo en el que unos pocos humanos supervivientes llevarán una vida más armoniosa.

Si se abandona el imaginario y sus visiones futuristas para mirar el mundo real y actual, otro tipo de literatura sobre el colapso conduce a la renuncia de los grandes relatos. Los antropólogos que estudian el tiempo de las catástrofes señalan, por un lado, que muchos pueblos, en particular los amerindios, ya han experimentado el colapso de su civilización y, por otro, que sobre las ruinas de un mundo florecen otros. Los grandes relatos sobre la Marcha de la Humanidad y el progreso técnico ceden el lugar ahora a pequeñas historias locales: por ejemplo, la historia de los buscadores de setas en los bosques californianos contada por Tsing (2015). Pequeñas historias atentas a los detalles de una situación local revelan otra forma de ver el colapso de la civilización al revelar alianzas inesperadas entre minorías étnicas, árboles y setas. Los hongos crecen en las zonas contaminadas por el accidente de Chernóbil, y quizás florezcan nuevas variedades de plantas y animales. Mientras que las catástrofes nucleares y el cambio climático amenazan el futuro de la humanidad, también abren oportunidades y un futuro para otras especies. Junto al tiempo de Cronos, el Titán que devoró a sus hijos para evitar ser asesinado por uno de ellos, hay lugar para el kairós, la oportunidad que hay que aprovechar el momento adecuado. El tiempo cronológico ya no es hegemónico. 


\section{Un recorrido paisajístico por la era del átomo}

Una segunda razón es que la energía nuclear puede llevar a una revisión de nuestras categorías temporales. Ello nos obliga a prestar atención al tiempo de las cosas. De hecho, por definición, los isótopos radiactivos que están en el origen de esta tecnología son activos. Demuestran que no podemos tratar las cosas como objetos y la materia como una masa inerte y pasiva, extendida en longitud, anchura y profundidad como decía Descartes. La materia es actuante, opera sobre el mundo y lo transforma; es un agente. Los elementos que lo componen y que se ponen en funcionamiento en los proyectos técnicos son todos muy individualizados, actúan y reaccionan ante el entorno. Son tanto sujetos como objetos.

Entre ellos, los elementos radiactivos tienen la particularidad de que son menos sustancias que procesos: proceso de emisión de partículas alfa o beta, de captura de electrones o neutrones, o de fisión en dos o más nucleidos. Sea cual sea su estabilidad, sea cual sea su vida media, que puede ser desproporcionada con respecto a la vida de las sociedades humanas, los radionucleidos siempre están cambiando, siempre se están convirtiendo. Espontáneamente se transmutan en otros radionucleidos, se convierten en otros durante el proceso de desintegración radiactiva. Es porque son puro devenir que ofrecen un caso ejemplar para subvertir la ontología de la ciencia y la filosofía modernas. El tiempo no es el contenedor universal e indiferente al cambio que imaginó Newton. El tiempo es inmanente a los seres, a las cosas animadas e inanimadas. Cada uno tiene un tiempo propio. Hay tantos tiempos y líneas de vida como cosas. Una multiplicidad de tiempos que cohabitan, se cruzan, se interfieren, y así dibujan paisajes más o menos densos, armoniosos, diversos y variados.

El tiempo propio de las cosas puestas en funcionamiento en la era del átomo se distingue por un segundo carácter. La vida media de los radioisótopos depende únicamente de las propiedades del núcleo de los átomos. Cada radioisótopo tiene su propia vida media que es el marcador de su identidad. Es constante, rigurosamente predecible porque no se ve afectado por el entorno químico. Es precisamente esta independencia de todos los demás tiempos la que ha permitido utilizar ciertos radioisótopos (como el carbono-14) como trazadores para datar con precisión rocas u otros materiales y determinar el tiempo cronológico de la Tierra. A diferencia de los radioisótopos, que proporcionan relojes absolutos, la 
De las eras a los paisajes. Una perspectiva Crítica Sobre la «ERA Del Átomo»: encuentro entre CTS y Filosofía de la TECNOLOGÍA

mayoría de los materiales, incluidos los seres vivos, tienen tiempos de vida que se ven afectados por el medio ambiente y diversos fenómenos.

De ahí una tercera razón por la que la energía nuclear exige otra concepción del tiempo, una no lineal. La singularidad de los radioisótopos crea una notable asimetría porque su proceso de desintegración, que depende únicamente de las leyes de la física nuclear, tiene la propiedad de causar mucho daño a las cosas que los rodean. Las desgarradoras imágenes de los cuerpos destrozados tras la bomba de Hiroshima son un testimonio de su violencia, pero esta no se limita a las explosiones. La radiación ejerce una violencia más silenciosa, invisible y sigilosa sobre las células vivas que se manifiesta años después de la exposición. Esta «violencia lenta» (Nixon, 2013), insidiosa porque se funde con el propio tiempo, llevó a la invención del concepto de desastre lento. La catástrofe ya no es entonces un acontecimiento que pueda superarse y remontarse erigiendo un lugar de memoria como el Museo de la Paz en Hiroshima (Knowles, 2014). La radiación es una memoria activa en el presente que coloniza el futuro y lo determina según una necesidad inexorable.

Como modelos de tiempo inmanente a las cosas, los radioisótopos imponen inmediatamente una multiplicidad de tiempos que desafía la división tradicional entre tiempo subjetivo y objetivo. Aún más, plantean una pregunta práctica y vital: ¿cómo componer esos tiempos múltiples? ¿Cómo hacer coexistir las trayectorias de estos radioelementos con las de los habitantes de los alrededores, los trabajadores y residentes de las centrales, las plantas y los animales cercanos a los paquetes de residuos radiactivos, y las futuras generaciones de personas vivas que dentro de cientos de miles de años seguirán expuestas a la lenta violencia de la radiación? La energía nuclear nos obliga a ir más allá del tiempo cronológico para pensar en el tiempo-paisaje como una asociación o composición de líneas temporales múltiples y heterogéneas. En cuanto asumimos una multiplicidad de tiempos inmanentes a las cosas, surge la cuestión de sus interacciones. Todas las innovaciones tecnológicas introducen nuevas entidades y nuevas temporalidades. Y más que celebrar una innovación disruptiva, o una enésima revolución industrial, deberíamos hacernos la pregunta: ¿qué tipo de paisaje temporal nos ofrece esto? ¿Cómo podemos evitar tensiones o incluso conflictos perjudiciales, por supuesto, pero también crear interacciones positivas? El éxito de una tecnología radica en la invención de interdependencias exitosas o ensamblajes que permiten componer un territorio, para usar los términos de Deleuze y Guattari (1980). La 
evolución biológica ha encontrado soluciones elegantes a este problema general. Hay muchos ejemplos de alianzas exitosas y sostenibles, como las micorrizas, una asociación simbiótica entre hongos y raíces de árboles, o la microbiota intestinal. Las miles de bacterias que colonizan los intestinos de los mamíferos tienen una vida de unos 20 minutos, mientras que la vida de sus huéspedes es de años o décadas.

Lograr alianzas entre tiempos heterogéneos es precisamente lo que no pudo conseguir la era del átomo. Durante años, la cuestión de los residuos se consideró secundaria, una externalidad que no debía obstaculizar el auge nuclear ni la era del átomo. La perspectiva es muy diferente si razonamos en términos de tiempopaisaje. Las dificultades en el manejo de los residuos nucleares provienen, en primer lugar, de la disparidad de escalas temporales. La vida media del Pu239, el plutonio fabricado desde los años 40 para fabricar armas nucleares, es de 24110 años, es decir, aproximadamente el doble de la duración del Holoceno, mientras que la vida media de los gobiernos nacionales responsables de la gestión de estos residuos es de 5 años. Además, la dificultad se hace insuperable debido a la toxicidad del Pu-239 para todas las formas de vida. Los productos de larga duración y alta actividad son tan peligrosos que no pueden incluirse en ninguna alianza. La contención parece ser la solución. Por ello, todos los esfuerzos están dirigidos a construir barreras protectoras duraderas para evitar el contacto tanto en el presente como en el futuro lejano. La otra solución evidente, particularmente impulsada por los ingenieros franceses, sería elreprocesamiento. Pero los resultados son limitados y en cualquier caso los productos reprocesados producen residuos altamente tóxicos e intratables. Hasta ahora es imposible adaptar la edad del átomo a los imperativos de la economía circular, a menos que se pase de la fisión a la fusión. Como estos elementos fueron creados y están aquí para quedarse, aunque todos los países decidan eliminar la energía nuclear, la era del átomo no terminará. 
De las eras a los paisajes. Una perspectiva Crítica Sobre la «ERA del Átomo»: encuentro entre CTS y Filosofía de la TECNOLOGía

\section{CONCLUSIÓN}

En conclusión, la energía nuclear, generalmente considerada como una nueva era, la edad del átomo, integrada en la larga Marcha de la Humanidad, invita a pensar en el tiempo en términos distintos a los cronológicos. Este estudio de caso es adecuado para ilustrar los beneficios mutuos de una asociación entre CTS y filosofía de las técnicas.

Por un lado, la historia y la filosofía de las técnicas orientadas a objetos ayudan a sacar la corriente CTS del yo de la ciencia, la tecnología y la sociedad que no va más allá del marco del pensamiento moderno. La imaginación sociotécnica refleja esencialmente las experiencias socioeconómicas humanas de la historia de una época. Conectar el imaginario con la materialidad de las cosas, quizás rejuveneciendo la inspiración de Bachelard, podría desarrollar estudios sobre la historia y el imaginario de las técnicas. Además, a pesar de la apertura que creó en su época, la distinción entre humanos y no humanos sigue siendo muy antropocéntrica. Como la categoría de bárbaros mezcló a todos los que no eran griegos, o la de goyim a todos los que no son judíos, equivale a rechazar la infinita variedad de actores de la historia en una clase indistinta. Si tratamos a todos los seres vivos y no vivos como agentes de la historia, debemos ir más allá y considerar que el pasado, el presente y el futuro se pueden captar tanto a través de las cosas como de nuestros marcos temporales. Reforzar la alianza entre CTS y la historia o filosofía de las técnicas.

Por otro lado, la corriente CTS parece un antídoto contra las tendencias altamente especulativas de las filosofías que privan a los humanos de su monopolio como sujetos al reducir el mundo a la condición de objeto. De hecho, las conclusiones de este análisis crítico del tiempo cronológico coinciden en parte con la renovación ontológica conocida como «realismo especulativo». Meillassoux (2006), por ejemplo, se pregunta cómo puede existir el tiempo en el universo antes que los sujetos humanos si se inscribe en el sujeto como una forma a priori de sensibilidad y anula el kantismo. Mientras que Meillassoux apunta a estar más allá del pensamiento, independientemente de cualquier correlación, la filosofía de Harman (2010), a partir de Heidegger, postula los objetos como realidad primaria y describe sus relaciones y permutaciones a diferentes escalas como formando la sustancia misma del cosmos. Aunque estos enfoques especulativos tienen el inmenso interés de filosofar sobre los objetos, no nos permiten plantear la cuestión práctica y política: ¿cómo hacer mundo con actores heterogéneos? 


\section{REFERENCIAS}

Adam, B. (1998). Timescapes of Modernity: The Environment and Invisible Hazards. Routledge.

Announcing the Bombing of Hiroshima. (1945, 6 de agosto). American Experience. https: / / www.pbs.org/wgbh/americanexperience / features/trumanhiroshima/

Austin, J. L. (1962). How to Do Things with Words. Clarendon Press.

Badash, L. (2009). A Nuclear Winter's Tale: Science and Politics in the 1980s. The MIT Press.

Baschet, J. (2018). Défaire la tyrannie du présent. Temporalités émergentes et futurs inédits. La Découverte.

Bensaude-Vincent, B. (2021). Temps-paysage: Pour une écologie des crises. Éditions Le Pommier.

Bijker, W. E.; Hughes, T. P.; Pinch, T. (eds.). (1987). The Social Construction of Technological Systems: New Directions in the Sociology and History of Technology. The MIT Press.

Bonneuil, C.; Fressoz, J. B. (2012). L'événement Anthropocène. La terre, l'histoire et nous. Seuil.

Bouton, C.; Huneman, P. (eds.). (2017). Time of Nature and the Nature of Time. Philosophical Perspectives of Time in Natural Sciences. Springer. https: / / doi.org/10.1007/978-3-319-53725-2

Brey, P. (2010). Philosophy of Technology after the Empirical Turn. Techné: Research in Philosophy and Technology, v. 14, n. 1, 36-48. https: / / doi.org / 10.5840 / techne20101416

Camus, A. (1945, 08 de Agosto). Éditorial. Combat.

Chakrabarty, D. (2009). The Climate of History: Four Theses. Critical Inquiry, v. 35, n. 2, 197-222. https:/ / doi.org/10.1086/596640

Charbonneau, B. (2014). An deux mille. En B. Charbonneau; J. Ellul, Nous sommes des révolutionnaires malgré nous (pp. 193-215). Seuil.

Conty, A. (2016). Who is to Interpret the Anthropocene? Nature and Culture in the Academy. La Deleuziana, n. 4, 19-44. 
De las eras a los paisajes. Una perspectiva Crítica Sobre la «ERA Del Átomo»: encuentro entre CTS y Filosofía de la TECNOLOGÍA

Crutzen, P. J.; Birks, J. (1982). The Atmosphere after a Nuclear War: Twilight at Noon. Ambio, v. 11, n. 2/3, 114-125.

Crutzen, P. J.; Stoermer, E. F. (2000). The “Anthropocene". Global Change Newsletter, n. $41,17-18$.

Danowski, D.; Viveiros de Castro, E. (2016). The Ends of the World. Polity.

Daston, L.; Galison, P. (2007). Objectivity. Zone Books.

Deleuze, G.; Guattari, F. (1980). Mille Plateaux. Capitalisme et schrizophrnie. Les Éditions de Minuit.

Descartes, R. (1637). Discours de la méthode. Leyde.

Descola, P. (2018a). Humain, trop humain. En R. Beau; C. Larrère. (dirs.). Penser l'Anthropocène (pp. 19-35). Presses de Science Po.

Descola, P. (2018b). Is the Anthropocene soluble in ontological pluralism? https: / / www.anthropocene-curriculum.org/ contribution/ anthropocenelecture-philippe-descola

Dörries, M. (2011). The Politics of Atmospheric Sciences: "Nuclear Winter" and Global Climate Change. Osiris, v. 26, n. 1, 198-223. https: / / doi.org/10.1086/661272

Edgerton, D. (2007). The Shock of the Old. Technology and Global History Since 1900. Oxford University Press.

Feenberg, A. (2000). From Essentialism to Constructivism: Philosophy of Technology at the Crossroads. En E. Higgs; A. Light; D. Strong (eds.), Technology and the Good Life? (pp. 294-315). University of Chicago Press.

Feenberg, A. (2003). Modernity Theory and Technology Studies: Reflections on Bridging the Gap. En T. J. Misa; P. Brey; A. Feenberg (eds.), Modernity and Technology (pp. 73-104). The MIT Press.

Foucault, M. (1966). Les mots et les choses. Une archéologie des scienes humaines. Éditions Gallimard.

Fressoz, J. B. (2014). Pour une histoire désorientée de l'énergie. En 25èmes Journées scientifiques de l'environnement. https:// hal.archives-ouvertes.fr / hal-00956441/ document 
Hacking, I. (1983). Representing and Intervening. Introductory Topics in the Philosophy of Natural Science. Cambridge University Press.

Haraway, D. J. (2007). When Species Meet. University of Minnesota Press.

Hård, M.; Jamison, A. (eds.). (1998). The Intellectual Appropriation of Technology: Discourses on Modernity, 1900-1939. The MIT Press.

Hård, M.; Jamison, A. (2005). Hubris and Hybrids. A Cultural History of Technology and Science. Routledge.

Harman, G. (2010). L'objet quadruple : Une métaphysique des choses après Heidegger. Presses Universitaires de France.

Hartog, F. (2015). Régimes d'historicité. Présentisme et expériences du temps. Points.

Hooft, G.; Vandoren, S. (2014). Time in Powers of Ten. World Scientific Publishing. https: / / doi.org/10.1142/8786

International Geophere-Biosphere Programme. (2015). Great Acceleration. http: / / www.igbp.net/globalchange/ greatacceleration.4.1b8ae20512db692f 2a680001630.html

Jasanoff, S.; Kim, S. H. (eds.). (2015). Dreamscapes of Modernity: Sociotechnical Imaginaries and the Fabrication of Power. University of Chicago Press.

Kant, I. (1784). Idées d'une histoire universelle au point de vue cosmopolitique. Bordas.

Knowles, S. G. (2014). Learning from Disaster? The History of Technology and the Future of Disaster Research. Technology and Culture, v. 55, n. 4, 773-784. https: / / doi.org/10.1353/ tech.2014.0110

Kuchinskaya, O. (2014). The Politics of Invisibility: Public Knowledge about Radiation Health Effects after Chernobyl. The MIT press.

Lakoff, G.; Johnson, M. (1980). Metaphors We Live by. University of Chicago Press.

Latour, B. (1987). Science in Action: How to Follow Scientists and Engineers through Society. Harvard University Press.

Latour, B. (1991). Nous n'avons jamais été modernes. Éditions La Découverte.

Latour, B. (1999). Politiques de la nature. Comment faire entrer les sciences en démocratie. Éditions La Découverte. 
De las eras a los paisajes. Una perspectiva Crítica Sobre la «ERA Del Átomo»: encuentro entre CTS y Filosofía de la TECNOLOGía

Latour, B. (2014). Agency at the Time of the Anthropocene. New Literary History, v. 45, n. 1, 1-18. https: / / doi.org/10.1353/nlh.2014.0003

Latour, B. (2017). Facing Gaia. Eight Lectures on the New Climatic Regime. Polity.

Lewis, S. L.; Maslin, M. A. (2015). Defining the Anthropocene. Nature, v. 519, 171180. https: / / doi.org/10.1038/nature14258

Linnér, B. O.; Wibeck, V. (2015). Dual high-stake emerging technologies: A review of the climate engineering research literature. WIREs Climate Change, v. 6, n. 2, 255-268. https: / / doi.org/10.1002/ wcc.333

Loeve, S.; Guchet, X.; Bensaude-Vincent, B. (eds). (2018). French Philosophy of Technology: Classical Readings and Contemporary Approaches. Springer.

Meillassoux, Q. (2006). Après la finitude. Essai sur la nécessité de la contingence. Éditions du Seuil.

Mol, A. (2002). The Body Multiple: Ontology in Medical Practice. Duke University Press.

Monastersky, R. (2015). Anthropocene. The Human Age. Nature, v. 519, 144-147. https: / / doi.org/10.1038/519144a

Moore, J. W. (ed.). (2016). Anthropocene or Capitalocene? Nature, History, and the Crisis of Capitalism. PM Press.

Mumford, L. (1946). Gentlemen: You are mad! The Saturday Review of Literature, v. 29 , n. 2, 5-6.

Nixon, R. (2013). Slow Violence and the Environmentalism of the Poor. Harvard University Press.

Pinault, M. (2000). Frédéric Joliot-Curie. Odile Jacob.

Pinch, T. J.; Bijker, W. E. (1984). The Social Construction of Facts and Artefacts: Or How the Sociology of Science and the Sociology of Technology might Benefit Each Other. Social Studies of Science, v. 14, n. 3, 399-441. https: / / doi.org/ 10.1177 / 030631284014003004

Sartre, J. P. (1945). Présentation. Les Temps Modernes, n. 1.

Serres, M. (1980). Le passage du Nord-Ouest. Les Éditions de Minuit.

Serres, M. (1990). Le contrat naturel. Éditions François Bourin. 
Serres, M. (1994). Atlas. Julliard.

Serres, M. (2019). Relire le relié. Éditions Le Pommier.

Singer, P. (2012). La libération animale. Payot.

Srinivasan, K.; Kasturirangan, R. (2016). Political ecology, development and human exceptionalism. Geoforum, v. 75, 125-128. https: / / doi.org/10.1016/j.geoforum.2016.07.011

Steffen, W.; Broadgate, W.; Deutsch, L.; Gaffney, O.; Ludwig, C. (2015). The trajectory of the Anthropocene: The Great Acceleration. The Anthropocene Review, v. 2, n. 1, 81-98. https:/ / doi.org/10.1177/2053019614564785

Tsing, A. L. (2015). The Mushroom at the End of the World. On the Possibility of Life in Capitalist Ruins. Princeton University Press.

Topçu, S. (2013). La France nucléaire. L'art de gouverner une technologie contestée. Seuil.

Truong, N. (2020, 4 de agosto). Le tournant écopolitique de la pensée française. Le Monde. https:/ / www.lemonde.fr/idees/article/2020/08/02/le-tournantecopolitique-de-la-pensee-francaise_6047969_3232.html

Verbeek, P. P. (2005). What Things Do: Philosophical Reflections on Technology, Agency, and Design. Penn State University Press.

Verbeek, P. P. (2008). Obstetric Ultrasound and the Technological Mediation of Morality: A Postphenomenological Analysis. Human Studies, v. 31, n. 1, 11-26. https: / / doi.org/10.1007/s10746-007-9079-0

Weart, S. (1992). From the Nuclear Frying Pan into the Global Fire. Bulletin of the Atomic Scientists, v. 48, n. 5, 18-27. https:// doi.org/10.1080/00963402.1992.11 456271

Zwigenberg, R. (2015). Never Again: Hiroshima, Auschwitz and the Politics of Commemoration. The Asia-Pacific Journal, v. 13, n. 3, 1-22. https: / / apjjf.org/-Ran-Zwigenberg/ 4252/ article.pdf 